\title{
Fatores relacionados à suscetibilidade da erosão em entressulcos sob condições de uso e manejo do solo
}

\author{
Flávio P. de Oliveira ${ }^{1}$, D iogo C. Buarque ${ }^{2}$, Ana C. Viero ${ }^{3}$, \\ Gustavo H. Merten ${ }^{2}$, Elemar A. Cassol ${ }^{4} \&$ Jean P. G. Minella ${ }^{5}$
}

\begin{abstract}
RESU M O
N este trabalho foram avaliados fatores relacionados com a suscetibilidade a erosão em entressulcos de um $\mathrm{N}$ eossolo Litólico submetido a diferentes intensidades de uso e manejo do solo. 0 experimento foi realizado em condições de laboratório, utilizando-se amostras deformadas de solo colocadas em parcelas experimentais $\left(0,23 \mathrm{~m}^{2}\right)$ e declividade de $0,09 \mathrm{~m} \mathrm{~m}^{-1}$. 0 delineamento experimental utilizado foi em blocos casualizados, em que foram aplicadas chuvas simuladas com intensidade de $100 \mathrm{~mm} \mathrm{~h}^{-1}$ para os seguintes tratamentos: (I) solo cultivado com fumo sob preparo convencional (PC); (II) solo cultivado com fumo sob plantio direto (PD) e (III) solo sob mata nativa (M N). Para avaliar a suscetibilidade a erosão em entressulcos utilizaram-se índices referentes à relação energia cinética total (chuva e escoamento) sobre perda de solo, taxa média de desagregação e índice de estabilidade de agregados, cujos resultados mostraram que os fatores relacionados com a suscetibilidade a erosão em entressulcos estão associados não apenas com características e propriedades que conferem coesividade ao solo, mas, também, com condicionantes que afetam a hidráulica do escoamento e, consequentemente, a fase de transporte dos sedimentos.
\end{abstract}

Palavras-chave: processos erosivos, erosão hídrica do solo, chuva simulada, variáveis hidráulicas do escoamento

\section{Factors influencing susceptibility to interrill soil erosion under different land use and management conditions}

\begin{abstract}
This study evaluated factors related to the suscetibility to the interrill soil erosion in an Entisol subjected to different degrees of soil use and management. The experiment was carried out under laboratory conditions using samples collected from tobacco fields and disturbed soil placed in erosion pans measuring $\left(0.23 \mathrm{~m}^{2}\right)$ with a slope of $0.09 \mathrm{~m} \mathrm{~m}^{-1}$. The experimental design was in randomized blocks. Simulated rainfall intensity of $100 \mathrm{~mm} \mathrm{~h}^{-1}$ was applied to the following treatments: (I) conventionally tilled soil; (II) no-till soil; and (III) native forest soil. Total kinetic energy (rainfall and runoff) to soil loss, average rate of detachment, and aggregate stability indexes were used to assess susceptibility to interrill erosion. Results show that the factors related with the susceptibility to interrill erosion are associated not just to the characteristics and properties that confer cohesivity to soil, but also to those factors that affect runoff hydraulics and therefore the sediment transport phase as well.
\end{abstract}

Key words: erosive processes, soil erosion, simulated rainfall, hydraulic variables of runoff

\footnotetext{
${ }^{1}$ DS/U FPB. Rodovia PB 079 - km 12, Cidade Universitária, CEP 58397-000, Areia, PB. E-mail: pereira@cca.ufpb.br 2 Instituto de Pesquisas Hidráulicas/ UFRGS. Av. Bento Gonçalves 9500, CEP 91501-970, Porto Alegre, RS. E-mail: diogo.buarque@gmail.com; merten@iph.ufrgs.br

${ }^{3}$ CPRM - Serviço Geológico do Brasil. CEP 90840-030, Porto Alegre, RS. E-mail: anaclaudia@pa.cprm.gov.br

${ }^{4}$ DS /UFRGS. Av. Bento Gonçalves 7712, CEP 91501-970, Porto Alegre, RS. E-mail: cassolea@orion.ufrgs.br

${ }^{5}$ D S /U FSM. Av. Roraima 1000, CEP 97105-900, Santa M aria, RS. E-mail: jminella@gmail.com
} 


\section{INTRODUÇÃO}

A erosão hídrica do solo envolve as etapas de desagregação, transporte e deposição de sedimentos. Meyer et al. (1975) sugeriram separar o processo de erosão hídrica em entressulcos e em sulcos. A erosão em entressulcos é aquela cujo agente erosivo responsável pela desagregação é a precipitação pluvial e o transporte ocorre por uma ação combinada do efeito salpicamento ("rain splash") e do escoamento difuso. A fonte de sedimentos no processo do entressulcos é constituída, basicamente, por materiais da superfície do solo. Já na erosão em sulcos o agente erosivo responsável pela desagregação e pelo transporte é o escoamento concentrado em que, sendo profundo o suficiente, protege a superfície do solo contra o impacto da gota da chuva enquanto a fonte de sedimentos constitui os horizontes superficiais e subsuperficiais (Bryan, 1987).

$\mathrm{Na}$ erosão em entressulcos, a maior parte do material desagregado é transferida para os sulco, através do escoamento difuso (Young \& Wiersma, 1973; Meyer et al., 1975). O escoamento difuso, devido à sua condição hidráulica de pouca espessura de lâmina de água em relação do elemento rugoso, tem parte de sua tensão cisalhante total dissipada em função da resistência de forma (Rauws \& Govers, 1988). Assim, a tensão cisalhante relacionada ao transporte de sedimentos e a desagregação do solo (tensão efetiva) são reduzidas (Abrahams \& Person, 1991). Por outro lado, o escoamento difuso sob ação da precipitação poderá ter sua capacidade de transporte aumentada quando algumas condições referentes à relação entre a altura de lâmina de água e o tamanho de gotas forem satisfeitas (Kinnell, 1991; 2003; 2005; 2006).

A transferência de sedimentos da região de onde esses sedimentos foram desagregados para a região dos sulcos, é um processo governado pelo princípio de que a carga de sedimentos a ser transportada é limitada ou pela disponibilidade de sedimentos ou, ainda, pela capacidade de transporte do escoamento difuso (Foster, 1982). Na maioria das vezes, o que limita este processo é a disponibilidade de sedimentos a serem transportados o que, por sua vez, é função das taxas de destacamento dos sedimentos da massa do solo (Toy et al., 2002). Desta forma, é oportuno considerar que a erosão em entressulcos é um processo dependente não apenas das condições envolvidas na fase de desagregação mas, também, pela capacidade de transporte do escoamento difuso. A este respeito, Kinnell (2005) tem proposto que o transporte de sedimentos na região em entressulcos pode ocorrer de três maneiras: a) transporte devido ao efeito de salpicamento (rain splash); b) transporte devido ao escoamento difuso induzido pela ação da precipitação e c) transporte pelo escoamento difuso sem ação de precipitação. De outra parte, tem-se verificado que a fase de transporte também afeta a taxa de desagregação de vez que partículas maiores se depositam durante o deslocamento e promovem a ação de proteção da superfície do solo contra a ação de desagregação dos sedimentos (Merten, et al., 2001; Kinnel, 2006).

Na etapa de desagregação a força erosiva é constituída pela energia cinética das gotas da chuva enquanto na resistência do solo atuam forças de natureza físico-química e biológica. A ação de resistência a desagregação está relacionada com fatores que conferem a estabilidade dos agregados do solo. Entre esses fatores se destacam: o teor da fração da argila na composição granulométrica do solo, o tipo de argila, a qualidade e o teor de matéria orgânica, a presença de óxidos de ferro e alumínio e a concentração e o tipo de cátions presentes na solução do solo (Grissenger, 1966; Line \& Meyer, 1989; Levy et al., 1994; Heil et al., 1997; Albuquerque et al., 2000; Nunes \& Cassol, 2008).

O uso e o manejo do solo alteram as forças de resistência a desagregação, especialmente as operações de preparo do solo que envolve uma mobilização do solo maior ou menor, exercendo grande influência sobre a estabilidade dos agregados. De maneira geral, sistemas que mobilizam menos o solo, como o cultivo mínimo e o plantio direto, promovem a elevação do carbono orgânico na superfície do solo sendo que esta condição, por sua vez, propicia um aumento da estabilidade dos agregados de tamanho maior (Melissa et al., 2006; Onweremadu et al., 2007; Razafimbelo et al., 2008; Simansky et al., 2008; Aratani et al., 2009; Jacobs et al., 2009; Veiga et al., 2009).

As práticas de correção do solo e de adubação também influenciam a maior ou a menor resistência do solo a desagregação. $\mathrm{O}$ uso de fertilizantes sintéticos e a adubação orgânica promovem uma produção maior de biomassa o que, por sua vez, favorece a maior estabilidade de agregados.porém em algumas situações o uso de corretivos ou fertilizantes pode promover a dispersão dos microagregados (Spera et al., 2008).

No sul do Brasil o cultivo do fumo tem, como característica, a grande mobilização do solo associado à aplicação de fertilizantes nitrogenado à base de nitrato de sódio $\left(\mathrm{NaNO}_{3}\right)$. Esta condição tem favorecido a erosão hídrica com consequente perda da capacidade produtiva dos solos e grande transferência de sedimentos para os rios (Merten \& Minella, 2005). Para reduzir esses efeitos tem sido difundido, nesta última década, o plantio direto para a cultura do fumo, alcançando resultados positivos em relação à redução da produção de sedimentos na escala de bacia (Minella et al., 2008). Sabe-se que a maior parte dos efeitos de redução da produção de sedimentos deve ser atribuída ao aumento da cobertura do solo proporcionado pelos cultivos conservacionistas. Entretanto, é notório o fato de que os sistemas conservacionistas atuam, também, na direção de uma estruturação melhor do solo, especialmente no que diz respeito a uma estabilidade maior de agregados. Assim, este trabalho teve como propósito investigar como os diferentes sistemas de uso e manejo do solo na cultura do fumo afetam o processo de erosão em entressulcos desconsiderando-se a cobertura do solo. Busca-se, quantificar o efeito do plantio direto do fumo na recuperação da condição original do solo em relação à suscetibilidade ao processo erosivo em entressulcos.

\section{Material E MÉTODOS}

O experimento foi realizado no Laboratório de Sedimentos do Instituto de Pesquisas Hidráulicas da Universidade Federal do Rio Grande do Sul (UFRGS), em parcelas experimentais com área útil de $0,23 \mathrm{~m}^{2}$, dimensões de $0,50 \mathrm{~m}$ por $0,46 \mathrm{~m} \mathrm{e}$ profundidade de $0,07 \mathrm{~m}$. O volume da parcela experimental foi 
preenchido com uma camada de $3 \mathrm{~cm}$ de espessura de areia e, sobre esta, uma tela de plástico com malha de $1 \mathrm{~mm}$, sobre a qual se colocou uma camada de solo com $4 \mathrm{~cm}$ de espessura, para uma densidade de empacotamento (Dep) de $1,2 \mathrm{Mg} \mathrm{m}^{-3}$. A declividade das parcelas experimentais foi de $0,09 \mathrm{~m} \mathrm{~m}^{-1}$.

O solo utilizado no presente estudo foi proveniente de uma bacia hidrográfica experimental localizada no município de Arvorezinha, região centro-norte do estado do Rio Grande do Sul, e contemplou uma das principais classes de solo identificadas na região, Neossolo Litólico distrófico típico (Streck et al., 2008). O clima da região, segundo classificação de Koppen, é do tipo $\mathrm{Cfb}$, subtropical úmido com verões quentes, inverno com geadas frequentes e precipitação bem distribuída ao ano (Minella et al., 2007).

Amostras de solo foram coletadas a campo na camada superficial de 0-20 cm de profundidade. Após secagem ao ar, foram destorroadas e peneiradas em malha de $8 \mathrm{~mm}$ de diâmetro. Posteriormente, já nas parcelas, foram umedecidas com antecedência de 1 hora, deixando o solo com umidade na capacidade de campo.

O delineamento experimental foi em blocos casualizados, nos quais foram aplicadas chuvas simuladas sobre as parcelas de erosão em entressulcos, com os seguintes tratamentos: (I) solo sob preparo convencional (PC), proveniente de área com cultivo de fumo com preparo do solo na forma tradicional, que consiste no revolvimento total do solo com arado de tração animal até uma profundidade média de $12 \mathrm{~cm}$. Associada a esta prática está vinculada a etapa de adubação da cultura, com uso de fertilizante nitrogenado na forma de nitrato de sódio; (II) solo sob plantio direto (PD) há 6 anos, característico de áreas com ausência de práticas mecânicas no solo e com utilização da cultura do fumo em sucessão com plantas de cobertura (aveia, aveia + ervilhaca ou aveia + nabo forrageiro) associada a manejo com herbicida, favorecendo a cobertura do solo pela presença de resíduos vegetais na superfície e (III) solo sob mata nativa (MN), proveniente de Floresta Ombrófila Mista (Araucária angustifólia e arbustos arborescentes) (FEPAM, RS, 2008).

\section{Análises físicas e químicas do solo}

Para cada tratamento foi coletada amostra de solo na profundidade de $0-20 \mathrm{~cm}$, acondicionadas em sacos plásticos e, posteriormente, secadas ao ar, destorroadas e peneiradas em malha de $2 \mathrm{~mm}$, para caracterização química e física (Tabelas 1 e 2).

Na caracterização química foram determinados: o $\mathrm{pH}\left(\mathrm{H}_{2} \mathrm{O}\right)$; $\mathrm{Ca}, \mathrm{Mg}$ e Al trocáveis; $\mathrm{K}$ e Na e MO, conforme metodologia descrita em Tedesco et al. (1995).

Em relação à análise física do solo foi realizada sua análise granulométrica pela distribuição de diâmetro de partículas
Tabela 2. Caracterização física dos tratamentos utilizados no experimento

\begin{tabular}{|c|c|c|c|c|}
\hline \multirow{2}{*}{ Tratamento } & Grosseira $^{(1)}$ & Fina $^{(2)}$ & Argila & \multirow{2}{*}{$\begin{array}{c}\mathbf{G F}^{(3)} \\
\%\end{array}$} \\
\hline & \multicolumn{3}{|c|}{$\mathrm{g} \mathrm{kg}^{-1}$} & \\
\hline Convencional & 45,0 & 292,0 & 663,0 & 47,6 \\
\hline Direto & 68,0 & 599,0 & 333,0 & 51,5 \\
\hline Mata nativa & 103,0 & 353,0 & 543,0 & 51,5 \\
\hline
\end{tabular}

(1) Frações granulométricas incluindo a areia muito grossa $(2-1 \mathrm{~mm})$, grossa $(1-0,5 \mathrm{~mm})$ e media $(0,5-0,25 \mathrm{~mm}) .{ }^{(2)}$ Frações granulométricas incluindo as frações areia fina $(0,25-0,10 \mathrm{~mm})$ e muito fina $(0,10-0,05 \mathrm{~mm})$ e silte $(0,05-0,002 \mathrm{~mm})$, conforme Sistema de Classificação Norte-Americano (USDA); (3) Grau de floculação

primárias, conforme o método da pipeta (EMBRAPA, 1997), usando-se hidróxido de sódio $(\mathrm{NaOH}-1 \mathrm{~N})$ como agente dispersante (Tabela 2). Para determinação da argila dispersa em água utilizou-se o mesmo procedimento da determinação da argila total porém sem uso do dispersante químico. A partir dos dados foi possível calcular o grau de floculação, obtido da seguinte forma:

$$
\mathrm{GF}=\frac{\operatorname{Arg}-\operatorname{Arg}_{\mathrm{H} 2 \mathrm{O}}}{\operatorname{Arg}} \times 100
$$

donde: GF é o grau de floculação (\%); Arg é a fração de argila dispersa em hidróxido de sódio - $\mathrm{NaOH}\left(\mathrm{g} \mathrm{kg}^{-1}\right)$ e $\operatorname{Arg}_{\mathrm{H} 2 \mathrm{O}}$ é a fração da argila dispersa em água $\left(\mathrm{g} \mathrm{kg}^{-1}\right)$.

\section{Distribuição de tamanho e estabilidade de agregados}

Adicionalmente, a coleta de solo utilizado nas parcelas experimentais, foi realizada nas amostras de solo, na profundidade de $0-20 \mathrm{~cm}$, para determinação da estabilidade de agregados.

A classificação por tamanho e a estabilidade dos agregados a úmido foram determinadas com base na metodologia descrita por Kemper \& Chepil (1965), modificada por Tisdall \& Oades (1979). Utilizando-se um aparelho de oscilação vertical semelhante ao de Yoder (1936), os agregados foram separados nas seguintes classes, com base no seu diâmetro: 10,0-4,76; 4,76-2,00; 2,00-1,00; 1,00-0,50 e 0,50-0,25; 0,25-0,105; 0,105-0,053 e $<0,053 \mathrm{~mm}$. Na análise da distribuição de tamanho de agregados do solo a seco a metodologia foi semelhante aquela utilizada para avaliar a estabilidade dos agregados a úmido exceto que foi utilizado um equipamento do tipo Produtest, da Soil Test, com tempo de oscilação de $1 \mathrm{~min}$.

Com os valores de DMP a úmido e a seco, foi calculado o índice de estabilidade de agregados (IEA).

\section{Aplicação das chuvas simuladas}

As chuvas simuladas em laboratório foram efetuadas utilizando-se um simulador de chuvas, semelhante ao descrito

Tabela 1. Caracterização química dos tratamentos utilizados no experimento

\begin{tabular}{|c|c|c|c|c|c|c|c|c|c|}
\hline \multirow{2}{*}{ Tratamento } & \multirow{2}{*}{$\begin{array}{c}\mathrm{pH} \\
\mathrm{H}_{2} \mathrm{O}\end{array}$} & $\mathrm{Ca}$ & $\mathrm{Mg}$ & \multirow{2}{*}{$\begin{array}{c}\mathrm{Na} \\
\mathrm{mg} \mathrm{dm}\end{array}$} & \multirow{2}{*}{$\begin{array}{c}\text { CTC }^{(1)} \\
\mathrm{cmol}_{\mathrm{c}} \mathrm{dm}^{-3}\end{array}$} & \multirow{2}{*}{$\begin{array}{l}M .0^{(2)} \\
\mathrm{g} \mathrm{dm}^{-3}\end{array}$} & \multicolumn{3}{|c|}{ Relações } \\
\hline & & \multicolumn{2}{|c|}{$\mathrm{cmol}_{\mathrm{c}} \mathrm{dm}^{-3}$} & & & & $\mathrm{Na} / \mathrm{CTC}$ & $\mathrm{Ca}+\mathrm{Mg} / \mathrm{CTC}$ & $\mathrm{Ca} / \mathrm{Mg}$ \\
\hline Convencional & 4,7 & 5,7 & 2,4 & 28 & 24,2 & 53,0 & $5,03 \times 10^{-4}$ & 0,33 & 2,4 \\
\hline Direto & 5,5 & 5,4 & 2,5 & 27 & 13,6 & 23,0 & $8,64 \times 10^{-4}$ & 0,58 & 2,2 \\
\hline Mata Nativa & 4,5 & 5,1 & 1,7 & 20 & 24,8 & 58,0 & $3,51 \times 10^{-4}$ & 0,27 & 3,0 \\
\hline
\end{tabular}


por Meyer \& Harmon (1979), com bico aspersor tipo Veejet 80100, situado a 2,7 $\mathrm{m}$ acima da superfície do solo, operando com pressão constante de $41 \mathrm{kPa}$ na extremidade de saída de água no bico, aferida por meio de um manômetro. As chuvas simuladas foram aplicadas com 3 repetições, cada uma com duração de 60 min e intensidade média de $100 \mathrm{~mm} \mathrm{~h}^{-1}$.

\section{Determinação das perdas de solo e água}

As taxas de perda de solo e a concentração de sedimentos foram obtidas pela pesagem do material coletado em potes de plástico com capacidade de $920 \mathrm{~mL}$, colocados na extremidade inferior da calha coletora; em seguida, foram acrescentados 5 $\mathrm{mL}$ de Sulfato de Alumínio e Potássio [ $\mathrm{KAl}\left(\mathrm{SO}_{4}\right)_{2} \cdot 12 \mathrm{H}_{2} \mathrm{O}$ ] a $5 \%$ para acelerar o processo de sedimentação; após $24 \mathrm{~h}$ o sobrenadante foi sifonado e o solo encaminhado para secagem em estufa, na temperatura de 50 a $60^{\circ} \mathrm{C}$; em seguida, os potes foram pesados com e sem o solo seco. A relação entre a massa de solo seco e a massa da mistura água-sedimento expressa a concentração de sedimentos em peso $\left(\mathrm{kg} \mathrm{kg}^{-1}\right)$.

\section{Cálculo da taxa de desagregação}

As perdas totais de solo em entressulcos foram obtidas pela expressão:

$$
\mathrm{PSi}=\frac{\Sigma \mathrm{QCt}}{\mathrm{A}}
$$

donde:

PSi - perda de solo em entressulcos, $\mathrm{kg} \mathrm{m}^{-2}$

Q - descarga total, $\mathrm{kg} \mathrm{s}^{-1}$

C - concentração de sedimentos na enxurrada, $\mathrm{kg} \mathrm{kg}^{-1}$

$\mathrm{t}$ - intervalo de tempo entre duas amostragens, $\mathrm{s}$

A - área da parcela, $\mathrm{m}^{2}$

As taxas de desagregação do solo em entressulcos foram determinadas conforme a expressão a seguir:

$$
\mathrm{Di}=\frac{\mathrm{Mss}}{\mathrm{A} \mathrm{Dc}}
$$

em que:

Di - taxa de desagregação do solo em entressulcos, $\mathrm{kg} \mathrm{m}^{-2} \mathrm{~s}^{-1}$

Mss - massa do solo seco desagregado, $\mathrm{kg}$

A - área da parcela, $\mathrm{m}^{2}$

Dc - duração da coleta, s.

\section{Cálculo da energia da chuva e do escoamento}

O tamanho das gotas foi determinado com uso do aparelho JOSS-WALDVOGEL Distrometer, obtendo-se alguns parâmetros relacionados às gotas de chuva (tamanho de gotas por classes, energia cinética, intensidade da chuva), conforme descrito por Semmelmann (1991) e Rockenbach \& Semmelmann (1998).

A altura de queda das gotas foi de aproximadamente 2,45 m e a pressão constante de 6 psi $(41 \mathrm{kPa})$ regulada através de manômetro. Os valores de velocidade terminal ( $v$ ) de todas as classes de gota $\left(\mathrm{cm} \mathrm{s}^{-1}\right)$ foram calculados através da equação derivada de Gunn \& Kinzer (1949) e das expressões ajustadas por Dingle \& Lee (1972), para os valores de $\mathrm{D}_{50} \leq 1,4 \mathrm{~mm} \mathrm{e}_{50}$ $>1,4 \mathrm{~mm}$, respectivamente:

$\mathrm{v}=-17,8951+448,94898 . \mathrm{d}_{\mathrm{i}}+16,3719 . \mathrm{d}_{\mathrm{i}}^{2}-45,9516 . \mathrm{d}_{\mathrm{i}}^{3}$

$v=24,166+448,8336 . d_{i}-75,6265 . d_{i}^{2}+4,2559 . d_{i}^{3}$

donde:

$\mathrm{d}_{\mathrm{i}} \quad$ - tamanho das gotas de cada classe $(\mathrm{mm})$

A energia cinética de cada gota $(\mathrm{J})$ foi calculada por meio da equação:

$$
\mathrm{Ec}=\frac{1}{2} \mathrm{~m} v^{2}
$$

donde:

$\mathrm{m}$ - massa, $\mathrm{kg}$, e $v$ a velocidade terminal da gota, $\mathrm{m} \mathrm{s}^{-1}$

A massa $m$ foi calculada através da distribuição das gotas obtidas pelo disdrômetro, por meio do diâmetro da gota. A energia cinética é expressa na unidade $\mathrm{J} \mathrm{m}^{-2} \mathrm{~h}^{-1}$ ou $\mathrm{MJ} \mathrm{ha}^{-1} \mathrm{~h}^{-1}$, obtida pela multiplicação da energia cinética pela intensidade e se dividindo pelo volume de gota. Este mesmo valor pode ser expresso na unidade $\mathrm{MJ} \mathrm{ha-1} \mathrm{mm}^{-1}$ dividindo o valor anterior pela intensidade da chuva. A EC total da chuva foi calculada somando-se a energia de todas as classes de gotas obtidas pelo espectro gerado pelo disdrômetro.

A energia do escoamento por unidade de área, é obtida diretamente por:

$$
\mathrm{EC}=\Omega \mathrm{t}
$$

em que: EC é a energia cinética do escoamento por unidade de área $\left(\mathrm{J} \mathrm{m}^{-2}\right.$ ou $\left.\mathrm{N} \mathrm{m}^{-1}\right)$, $\Omega$ é a potência do escoamento por unidade de área $\left(\mathrm{N} \mathrm{m}^{-1} \mathrm{~s}^{-1}\right.$ ou $\mathrm{J} \mathrm{s}^{-1} \mathrm{~m}^{-2}$ ou Watt $\left.\mathrm{m}^{-2}\right)$ e $\mathrm{t}$ é o tempo de duração da chuva (s).

A potência do escoamento por unidade de área $(\Omega)$ é definida por Rose (2004) pela expressão:

$$
\Omega=\tau \mathrm{V}
$$

donde:

$\tau$ é a tensão cisalhante $\left(\mathrm{N} \mathrm{m}^{-2}\right)$ e $\mathrm{V}$ é a velocidade do escoamento superficial $\left(\mathrm{m} \mathrm{s}^{-1}\right)$.

\section{Características hidráulicas do escoamento superficial}

A altura da lâmina do escoamento foi determinada através da equação derivada por Woolhiser \& Liggett (1967) e Singh (1983) para fluxo em um plano sob chuva de duração finita:

$$
\mathrm{h}=\frac{\mathrm{q}}{\mathrm{V}}
$$

sendo:

h - altura da lâmina de escoamento, $\mathrm{m}$

q - descarga líquida total por unidade de largura, $\mathrm{m}^{2} \mathrm{~s}^{-1}$ 
$\mathrm{V}$ - velocidade do escoamento, $\mathrm{m} \mathrm{s}^{-1}$

A descarga líquida (q) por unidade de largura, em $\mathrm{m}^{2} \mathrm{~s}^{-1}$, foi determinada a partir das coletas de enxurrada em potes plásticos coletados na extremidade da calha coletora, durante tempo cronometrado, e dividido pela largura da parcela. A velocidade superficial do escoamento (V) foi determinada relacionandose a distância entre dois pontos fixos na parcela e o tempo gasto para que um corante azul de metileno percorresse essa distância. Referidas determinações foram realizadas em intervalos de cinco minutos, a partir da formação da lâmina de escoamento. As velocidades médias do escoamento (V) foram definidas pelo produto das velocidades superficiais do escoamento por um fator de correção $(\alpha=2 / 3)$, conforme Katz et al. (1995).

O número de Reynolds foi obtido por meio da equação (Simons \& Senturk, 1992):

$$
\operatorname{Re}=\frac{\mathrm{Vh}}{\mathrm{v}}
$$

donde:

Re - número de Reynolds (adimensional)

$v \quad$ - viscosidade cinemática da água $\left(\mathrm{m}^{2} \mathrm{~s}^{-1}\right)$

O número de Froude foi obtido com a equação (Simons \& Senturk, 1992):

$$
\mathrm{Fr}=\frac{\mathrm{V}}{\sqrt{\mathrm{gh}}}
$$

sendo:

Fr - número de Froude, adimensional e g a aceleração da gravidade, $\mathrm{m} \mathrm{s}^{-2}$

Obteve-se o fator perda de carga (f) através da seguinte equação (Chow, 1959):

$$
f=\frac{8 \mathrm{ghS}}{\mathrm{V}^{2}}
$$

em que:

g - se refere à aceleração da gravidade, $\mathrm{m} \mathrm{s}^{-2}$

$\mathrm{h}$ - altura da lâmina de escoamento, $\mathrm{m}$

$\mathrm{S}$ - inclinação do declive, $\mathrm{m} \mathrm{m}^{-1}$.

\section{Análise dos dados}

Os resultados foram submetidos a análise de variância e, quando houve diferença significativa, compararam-se, então, as médias, pelo teste de Tukey $(\mathrm{p}<0,05)$. Utilizou-se o programa estatístico SAEG (SAEG, 2000).

\section{RESULTADOS E DISCUSSÃO}

Neste estudo a suscetibilidade da erosão em entressulcos foi analisada através da combinação de indicadores representados pela relação energia cinética total sobre a perda de solo total (EC/PS), pela taxa de desagregação (Di) e pelo índice de estabilidade de agregados (IEA), conforme a Tabela 3; os dois primeiros indicadores contemplam as etapas da desagregação e do transporte que ocorre na erosão em entressulcos, enquanto o IEA contempla apenas a fase de desagregação.

\section{Taxa de desagregação do solo (Di)}

Ainda que o fator Di seja denominado taxa de desagregação em entressulcos, de fato este fator representa a combinação do processo de destacamento das partículas da massa do solo e do deslocamento dos sedimentos, através do salpicamento e escoamento laminar difuso. Neste trabalho a taxa de desagregação em entressulcos (Di) foi avaliada através da quantificação dos sedimentos que foram desagregados e transportados na maior dimensão da parcela $(0,50 \mathrm{~m})$ que esteve orientada no sentido do declive até uma calha coletora.

As forças erosivas que estão envolvidas na determinação da Di são representadas pela energia cinética da chuva simulada (intensidade de $100 \mathrm{~mm} \mathrm{~h}^{-1}$ ) e do escoamento difuso caracterizado como laminar subcrítico, com número de Reynolds variando de 9,9 a 12,0 e Froude de 0,31 a 0,80 para os tratamentos MN e PC, respectivamente (Tabela 4). Os valores de $\operatorname{Re}<500$ e Fr $<1$ permitem inferir que ocorreu um escoamento laminar característico da erosão em entressulcos; já as forças de resistência ao destacamento e ao transporte do solo estão relacionadas com a coesividade e com o tamanho das partículas ou unidades estruturais que formam o solo.

Na Figura 1 são apresentados os valores do fator Di distribuídos no tempo para os três tratamentos avaliados. Observa-se que a taxa de desagregação variou durante o período inicial da chuva e os tratamentos PC e PD apresentaram redução nas taxas de $\mathrm{Di}$; logo a seguir aumentaram, até atingir um valor máximo tornando constantes até o período final da aplicação da chuva. Em termos relativos, os valores mais elevados de Di ocorreram para o tratamento PC e os menores para a MN. O

\begin{tabular}{|c|c|c|c|c|c|c|}
\hline \multirow[b]{2}{*}{ Tratamento } & \multirow[b]{2}{*}{$\begin{array}{l}\text { EC Total } \\
\text { J } \mathrm{m}^{-2} \mathrm{~h}^{-1}\end{array}$} & \multirow[b]{2}{*}{$\begin{array}{l}\text { Perda de solo } \\
\mathrm{kg} \mathrm{m}^{-2}\end{array}$} & \multicolumn{2}{|c|}{ Relação } & \multirow[b]{2}{*}{$\begin{array}{l}\text { Di médio(2) } \\
\mathrm{kg} \mathrm{m}^{-2} \mathrm{~s}^{-1}\end{array}$} & \multirow[b]{2}{*}{ IEA $A^{(3)}$} \\
\hline & & & $\begin{array}{l}\text { EC total/Perda de solo } \\
\qquad \mathrm{kJ} \mathrm{kg}^{-1} \mathrm{~h}^{-1}\end{array}$ & $\begin{array}{l}\text { Perda de Solo/EC total } \\
\qquad \mathrm{g} \mathrm{h} \mathrm{J}^{-1}\end{array}$ & & \\
\hline Convencional & 1935,2 a & $0,182 a$ & $10,632 b$ & $0,094 \mathrm{a}$ & $6,13 \times 10^{-4} \mathrm{a}$ & $0,70 \mathrm{~b}$ \\
\hline Direto & 1908,6 b & $0,160 \mathrm{a}$ & $11,928 \mathrm{~b}$ & $0,083 a$ & $5,02 \times 10^{-4} a$ & $0,89 \mathrm{a}$ \\
\hline Mata nativa & 1905,6 b & $0,081 \mathrm{a}$ & $23,525 a$ & $0,042 b$ & $3,61 \times 10^{-4} \mathrm{a}$ & $0,90 \mathrm{a}$ \\
\hline
\end{tabular}

Tabela 3. Valores médios da energia cinética $(\mathrm{EC})$ total, perda de solo, taxa média de desagregação e índice de estabilidade de agregados

(1) Energia cinética da chuva simulada (1763 $\left.\mathrm{J} \mathrm{m}^{-2} \mathrm{~h}^{-1}\right)$ mais a do escoamento difuso, (2) Taxa de desagregação de solo em entressulcos; ${ }^{(3)}$ Índice de estabilidade de agregados. Médias seguidas de mesmas letras, na coluna, não diferem entre si pelo teste de Tukey a $5 \%$ 
Tabela 4. Caracterização da hidráulica do escoamento para os diferentes tratamentos

\begin{tabular}{|c|c|c|c|c|c|c|c|c|c|c|c|}
\hline \multirow{2}{*}{ Tratamento } & $q^{(1)}$ & $\mathbf{V}^{(2)}$ & $h^{(3)}$ & $\operatorname{Re}^{(4)}$ & $\mathrm{Fr}^{(5)}$ & $f^{(6)}$ & \multirow{2}{*}{$\begin{array}{c}E C_{c s}^{(7)} \\
J m^{-2} h^{-1}\end{array}$} & \multirow{2}{*}{$\begin{array}{c}E C_{\text {esc. }}{ }^{(8)} \\
J m^{-2} h^{-1}\end{array}$} & \multirow{2}{*}{$\begin{array}{l}D_{50}{ }^{(9)} \\
\mathrm{mm}\end{array}$} & \multirow{2}{*}{$\begin{array}{c}\operatorname{CSS}_{\text {med }}^{(10)} \\
\mathrm{g} \mathrm{L}^{-1}\end{array}$} & \multirow{2}{*}{$\begin{array}{c}\text { CSS }_{\text {má }}^{(11)} \\
\mathrm{gL}^{-1}\end{array}$} \\
\hline & $m^{2} s^{-1}$ & $\mathrm{~m} \mathrm{~s}^{-1}$ & m & \multicolumn{3}{|c|}{ Admensional } & & & & & \\
\hline Conv & $1,25 \cdot 10^{-5} \mathrm{a}$ & $0,043 a$ & $0,00029 b$ & ב 120 & $0,80 a$ & $1,1 \mathrm{~b}$ & $1763 a$ & $172,2 \mathrm{a}$ & $0,037 a$ & $66,5 a$ & $91,3 \mathrm{a}$ \\
\hline Direto & $1,05.10^{-5} \mathrm{a}$ & $0,038 a b$ & $0,00028 b$ & & 0,7 & 1, & 17 & & 0,0 & & $83,0 \mathrm{~b}$ \\
\hline Mata nativa & $1,03 \cdot 10^{-5} a$ & $0,020 \mathrm{~b}$ & $0,00052 \mathrm{a}$ & $9,9 \mathrm{~b}$ & $0,31 \mathrm{c}$ & $13,8 \mathrm{a}$ & $1763 a$ & $142,6 \mathrm{~b}$ & $0,047 \mathrm{~b}$ & $50,0 \mathrm{~b}$ & $70,4 \mathrm{c}$ \\
\hline
\end{tabular}

${ }^{1)}$ Descarga líquida por unidade de largura; ${ }^{(2)}$ velocidade média; ${ }^{(3)}$ altura da lâmina de escoamento; ${ }^{(4)}$ número de Reynolds; ${ }^{(5)}$ número de Froude; ${ }^{(6)}$ fator de perda de carga; ${ }^{(7)(8)}$ energia cinética da chuva simulada e do escoamento difuso; ${ }^{(9)}$ diâmetro médio da partícula; ${ }^{(10)(11)}$ concentração de sedimento em suspensão média e máxima. Médias seguidas de mesmas letras, na coluna, não diferem entre si pelo teste de Tukey a $5 \%$

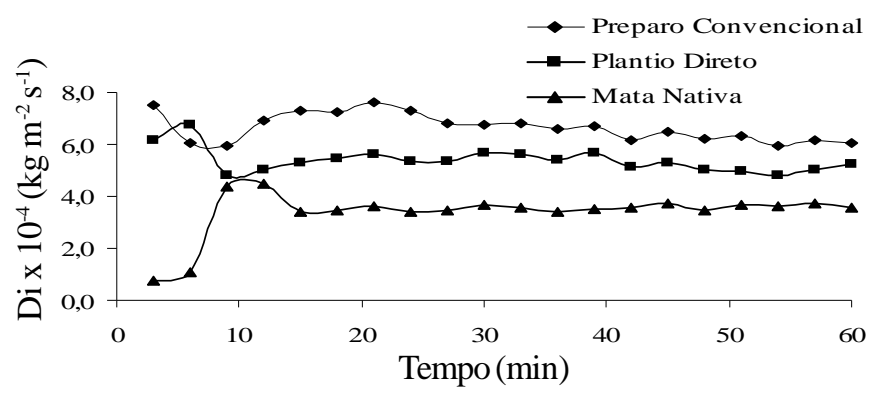

Figura 1. Taxas médias de desagregação do solo em entressulcos ( $\mathrm{Di})$, entre os tratamentos estudados, para os testes realizados em condições de laboratório

tratamento PD apresentou valores intermediários entre o PC e $\mathrm{MN}$, porém mais próximos dos valores do PC.

É muito provável que os valores decrescentes de Di no início da chuva estejam relacionados com a pronta disponibilidade de materiais facilmente destacáveis e transportáveis, presentes nos tratamentos PC e PD e que se esgotaram rapidamente (Figura 1). No instante posterior a esse decréscimo Di voltou a crescer até o momento em que as taxas de destacamento de solo se ajustaram com a capacidade de transporte de sedimentos.

Importante considerar que as diferenças observadas entre os tratamentos são decorrentes apenas das condições relativas da resistência do solo em relação ao destacamento e ao transporte, uma vez que todos os tratamentos se encontravam com as mesmas condições, ou seja, sem cobertura, com a mesma umidade antecedente ( $65 \%$ da umidade de saturação), a mesma densidade de empacotamento $\left(1,2 \mathrm{Mg} \mathrm{m}^{-3}\right)$ e com o mesmo declive $\left(0,09 \mathrm{~m} \mathrm{~m}^{-1}\right)$.

$\mathrm{Na}$ Tabela 3 se apresenta o resumo dos valores da energia cinética total (chuva mais escoamento difuso), da relação energia sobre perda de solo total, da taxa média de Di e da condição de resistência ao destacamento, representada pelo índice de estabilidade de agregados (IEA). De acordo com essa tabela, os valores EC totais calculados não apresentaram diferenças significativas entre os tratamentos PD e MN porém para o tratamento PC essa energia foi superior, diferindo estatisticamente da dos demais tratamentos, em função da maior EC do escoamento.

Quanto à relação energia sobre perda de solo total, verificase que o tratamento $\mathrm{MN}$ diferiu significativamente dos demais tratamentos, necessitando do dobro da energia para desagregar e transportar a mesma quantidade de solo. Esta condição sugere que, inicialmente, a suscetibilidade a erosão entressulcos do tratamento MN é menor que os tratamentos PC e PD e que entre esses dois tratamentos a suscetibilidade a erosão entressulcos seria semelhante; já com relação ao Di médio, os tratamentos não diferem entre si enquanto para o IEA o tratamento mata nativa (MN), juntamente com o plantio direto (PD), apresentou uma condição de agregação mais estável quando comparado com o sistema PC.

$\mathrm{O}$ fato dos tratamentos $\mathrm{MN}$ e $\mathrm{PD}$ não diferirem entre si pelo IEA enquanto pela relação de energia sobre perda de solo a MN tenha necessitado do dobro da energia para desagregar a mesma quantidade de solo, sugere que os fatores relacionados com a resistência a erosão em entressulcos estariam também relacionados com a fase de transporte.

\section{Análise da resistência do solo ao processo de desagregação através do IEA}

As características químicas e físicas do solo apresentadas nas Tabelas 1 e 2 auxiliam na interpretação dos resultados referentes às diferenças entre os tratamentos observados pelo IEA. Solos com maiores teor de argila e MO são mais resistentes à desagregação(Oades, 1988; Morgan, 1995). Porém, de acordo com as informações das Tabelas 1 e 2, os valores de $\mathrm{MO}$ e de argila verificados nos tratamentos $\mathrm{PC}$ e MN foram semelhantes entre si e diferentes do $\mathrm{PD}$, que apresentando valor de $\mathrm{MO}$ e de argila inferiores aos desses tratamentos. Desta forma, os maiores valores de $\mathrm{MO}$ e de argila verificados no tratamento MN explicam, em parte, a maior estabilidade de agregados para esse tratamento,, mas não é o caso do $\mathrm{PD}$, que apresentou menores valores de $\mathrm{MO}$ e de argila. Da mesma forma, verificase que o tratamento PD apresentou aproximadamente $60 \%$ da sua textura formada por material das frações silte e areia fina (Tabela 2), que são justamente aquelas frações consideradas mais facilmente erodíveis devido à pouca coesividade e facilidade de transporte dessas partículas.

Outro fator, também importante, relacionado com a resistência ao destacamento do solo, consiste no grau de floculação das argilas (Reichert \& Norton, 1995). Sabe-se que, quanto maior o grau de floculação das argilas menor será sua tendência à desagregação dos microagregados (base da cadeia hierárquica que forma os agregados) formados, basicamente, por argila floculada estabilizada por agentes cimentantes, como MO, óxidos de Fe e Al (Hillel, 2003). Neste caso e de acordo com a Tabela 2, o grau de floculação entre os três tratamentos foi semelhante, o que também não contribui para explicar as diferenças encontradas no IEA para os três tratamentos.

A presença dos cátions trocáveis na CTC do solo, especialmente do $\mathrm{Na}^{+}, \mathrm{Ca}^{++} \mathrm{e} \mathrm{Mg}^{++}$, exerce papel importante no processo de floculação e na dispersão das argilas. No caso dos solos utilizados neste estudo sabe-se que o $\mathrm{Na}^{+}$está presente no fertilizante empregado pelos fumicultores por meio 
de adubação nitrogenada com nitrato de sódio. Verificou-se, então, a influência desses três cátions sobre os processos de dispersão e floculação através das relações entre cátions e a CTC do solo. De acordo com essas relações (Tabela 1) verificase que a participação do $\mathrm{Na}^{+}$no complexo de troca de cátions se encontram várias ordens de grandeza inferior ao do $\mathrm{Ca}^{++}+$ $\mathrm{Mg}^{++}$. Segundo Davis et al. (2009), solos com problemas de dispersão de $\mathrm{Na}^{+}$normalmente apresentam a relação $\mathrm{Na}^{+} / \mathrm{CTC}$ superiores a $15 \%$. Outro aspecto relevante observado através

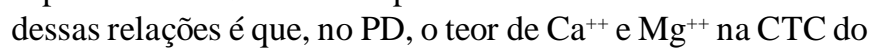
solo é praticamente o dobro do observado nos outros dois tratamentos.

A presença do cátion $\mathrm{Ca}^{++}$no complexo de troca e na solução do solo, quando hidratado, condiciona a formação de complexos de esfera interna cujo raio de hidratação é pequeno e, com isso, ocorre a compressão da dupla camada difusa, favorecendo a aproximação das argilas e o processo de floculação. Além da saturação de $\mathrm{Ca}^{++}$e $\mathrm{Mg}^{++}$, o grau de floculação e dispersão também está relacionado com as relações entre o $\mathrm{Ca}^{++}$e o $\mathrm{Mg}^{++}$. Segundo Bohn et al. (1995) e Dontsova \& Norton (2001) relações em que o $\mathrm{Ca}^{++}$é superior ao $\mathrm{Mg}^{++}$favorecem o processo de floculação uma vez que o $\mathrm{Mg}^{++}$tem seu raio de hidratação maior que o $\mathrm{Ca}^{++}$. De acordo com a Tabela 1 a relação entre $\mathrm{Ca}^{++}$e $\mathrm{Mg}^{++}$encontra-se na ordem de 2:1 para os tratamentos PC e PD e 3:1 para a $\mathrm{MN}$, indicando que, para todos os tratamentos, as relações $\mathrm{Ca}^{++}$e $\mathrm{Mg}^{++}$foram favoráveis ao processo de floculação das argilas, principalmente no MN.

Então, uma hipótese possível de ser levantada para explicar o maior IEA encontrado para o PD em comparação ao PC, já que o teor de $\mathrm{MO}$ e argila desse tratamento foi inferior aos demais, poderia estar relacionada a dois possíveis aspectos: à condição natural de adensamento do solo quando submetido

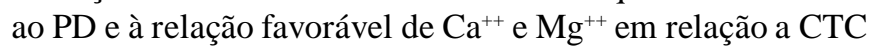
do solo.

Outro aspecto que deve ser considerado está relacionado ao método utilizado para determinar o IEA. Sabe-se que agregados que possuem maior densidade tendem a apresentar condição de maior estabilidade, quando esta é avaliada através do método do tamisamento úmido e seco. Assim, ainda que neste estudo tenha sido utilizado solo com estrutura deformada e com uma mesma densidade de empacotamento $\left(1,2 \mathrm{Mg} \mathrm{m}^{-3}\right)$ nos três tratamentos, o maior adensamento do $\mathrm{PD}$ pode persistir entre os agregados maiores. Mello (2006), estudando atributos do solo submetido a diferentes sistemas de manejo para o mesmo local onde foram coletados os solos deste estudo, verificou uma densidade de solo para a camada de $0-17 \mathrm{~cm}$ de 1,09, 1,23 e 0,93 $\mathrm{Mg} \mathrm{m}^{-3}$ para o PC, PD e MN, respectivamente.

Desta forma, presume-se que a combinação do efeito do adensamento com a maior presença de $\mathrm{Ca}^{++}$no complexo de trocas tenha condicionado um IEA semelhante entre os tratamentos PD e MN.

\section{Análise da resistência do solo à fase de transporte da erosão em entressulcos}

Em relação à fase de transporte de sedimentos na erosão em entressulcos, ela ocorre não apenas pela ação de salpicamento da gota da chuva (efeito "splash"), mas principalmente pelo escoamento superficial difuso que ocorre na região de entressulcos (Meyer et al., 1975). Em geral, o escoamento difuso apresenta características hidráulicas do tipo laminar subcrítico, conforme se constata em Reynolds e Froude, na Tabela 4; no entanto e dependendo da relação entre a altura da lâmina de água e o diâmetro das gotas da chuva, esse tipo de escoamento pode atenuar as taxas de desagregação provocadas pelo impacto das gotas da chuva e, ao mesmo tempo, aumentar a capacidade de transporte do escoamento devido a um aumento de momento causado pela turbulência (Savat, 1979). De acordo com Kinnel (1991), este aumento de turbulência é capaz de ocorrer quando a altura de lâmina do escoamento difuso é de aproximadamente $1,5 \mathrm{vez}$ menor que o diâmetro das gotas da chuva. Desta maneira, quando se analisa a taxa de desagregação (Di) é importante considerar que ela é governada tanto pela disponibilidade de sedimentos desagregados como pela capacidade do escoamento de transportar os sedimentos desagregados (Toy et al., 2002).

Nas Figuras 2A, 2B e 2C tem-se os gráficos que representam a variação temporal do escoamento superficial e da concentração de sedimentos em suspensão para os tratamentos PC, PD e MN, respectivamente. Verifica-se, na Figura 2A (tratamento PC) que as taxas do escoamento superficial já se iniciaram próximas a um valor constante, porém mais elevadas se comparadas com as taxas dos demais tratamentos. Os valores crescentes do escoamento representam a fase em que o solo ainda não se encontrava completamente saturado ou, então, quando ainda não havia ocorrido a formação do selo superficial.

A caracterização da hidráulica do escoamento para os diferentes tratamentos é apresentada na Tabela 4. De acordo com os resultados, não houve diferenças significativas da descarga líquida por unidade de largura entre os tratamentos; verificou-se, porém, um valor absoluto maior, no PC $\left(1,25.10^{5}\right.$ $\left.\mathrm{m}^{2} \mathrm{~s}^{-1}\right)$ e menor valor na $\mathrm{MN}\left(1,03 \cdot 10^{5} \mathrm{~m}^{2} \mathrm{~s}^{-1}\right)$. Além deste aspecto verrificou-se, através do número de $\operatorname{Re}(<500)$, que o regime do escoamento para todos os tratamentos foi laminar; apesar disto, é importante considerar, conforme já comentado, que a energia do escoamento também é afetada pela energia do impacto da gota de chuva, a qual pode condicionar um aumento da turbulência. No caso deste experimento a relação entre altura da lâmina do escoamento (Tabela 4$)$ e o diâmetro da gota $\left(\mathrm{D}_{50}=\right.$ $0,6 \mathrm{~mm}$ ) apresentou valores de 0,48, 0,46 e 1,2 para os tratamentos PC, PD e MN, respectivamente.

Através da Tabela 4 pode-se verificar, também, diferenças significativas na hidráulica do escoamento para a velocidade (V) e para a altura do escoamento (h), sendo essas diferenças condicionadas pela rugosidade superficial quantificada através da maior perda de carga $(f)$. Conforme se pode constatar na Tabela 4, a maior $f$ foi verificada para o tratamento MN. A perda de carga $(f)$ em termos da hidráulica do escoamento representa a parcela da energia relacionada com as forças de resistência ao deslocamento do escoamento superficial, representadas pela rugosidade superficial.

Em relação à EC do escoamento verifica-se, através da Tabela 4, um valor ligeiramente superior para o tratamento PC quando comparado com o PD e a MN. A maior energia do escoamento quantificada para o PC resultou em maior capacidade de transporte de sedimentos, passível de ser constatada pelo valor mais elevado da CSS $_{\text {máx }}$ verificada para o tratamento PC $\left(91,3 \mathrm{~g} \mathrm{~L}^{-1}\right)$ 
A.

B.
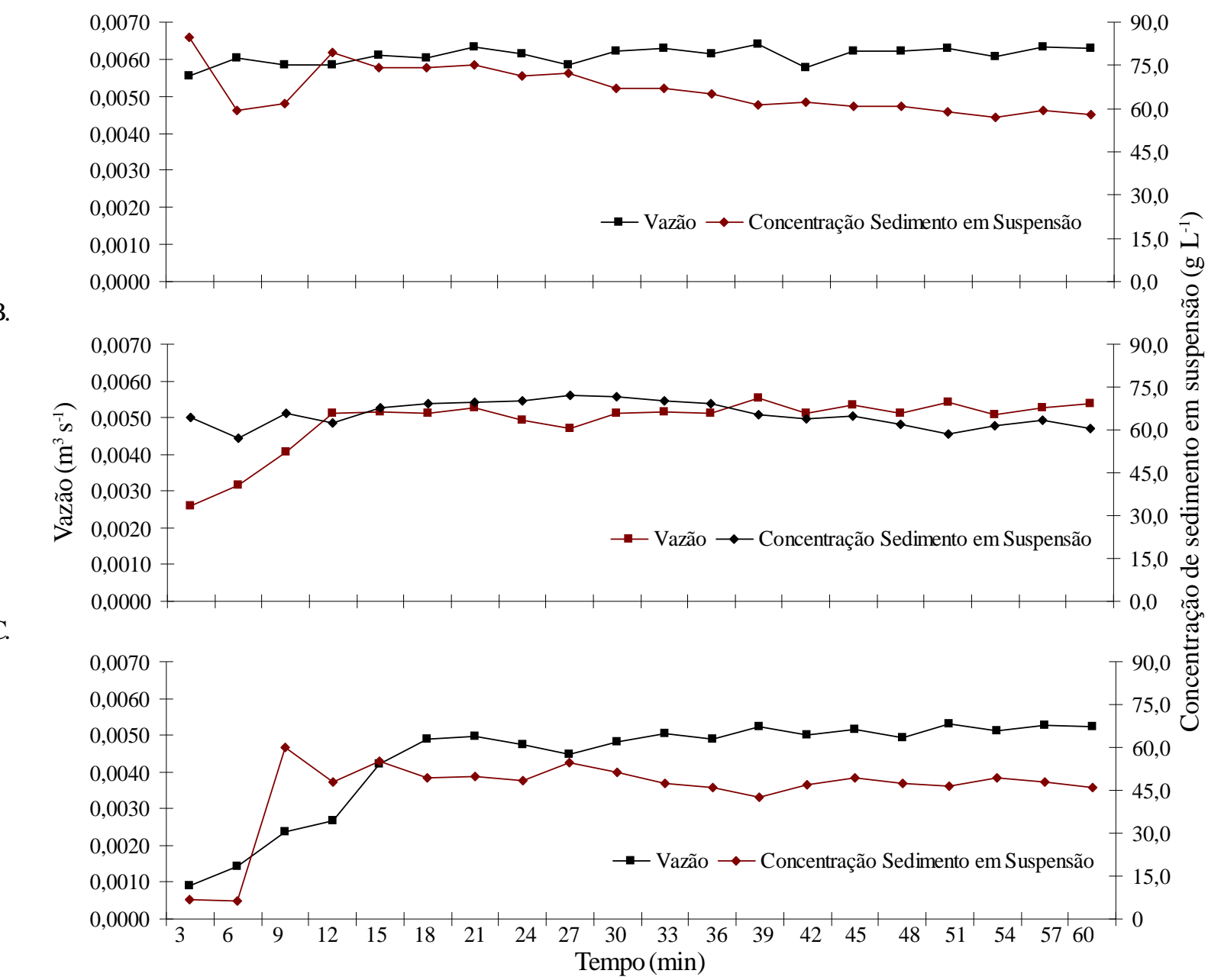

Figura 2. Distribuição temporal do escoamento superficial e concentração de sedimentos em suspensão avaliada para os sistemas de preparo de solo convencional (A); plantio direto (B); e mata nativa (C)

quando comparada com o PD $\left(82,9 \mathrm{~g} \mathrm{~L}^{-1}\right)$ e a $\mathrm{MN}\left(70,4 \mathrm{~g} \mathrm{~L}^{-1}\right)$. Verifica-se também que o tamanho dos sedimentos transportados foi muito semelhante para os três tratamentos e corresponde à fração silte grosso $\left(\mathrm{D}_{50}\right.$ de 0,037 a $\left.0,047 \mathrm{~mm}\right)$ (Tabela 4). Esta condição ocorreu independentemente da textura do solo e, segundo Morgan (1995), corresponde ao tamanho de partícula que, normalmente, é desagregada pela ação do impacto das gotas da chuva.

De acordo com a Tabela 3, a taxa de desagregação média (Di) entre tratamentos não se diferenciou entre si. Por outro lado, através do IEA verificou-se que o tratamento PC se diferenciou dos tratamentos PD e MN, ainda que esses últimos não se tenham diferenciado entre si. Aparentemente, tais resultados demonstram alguma inconsistência. Considerando que a relação energia sobre perda de solo tenha sido semelhante para o PC e o PD e que o tratamento MN necessitou do dobro da energia para erodir a mesma quantidade de solo, seria de se esperar que tanto a Di como o IEA (Tabela 3) apresentassem a mesma tendência observada para a relação energia por perda de solo. Especialmente a falta de consistência entre a relação de energia sobre perda de solo total com o IEA permite concluir que a Di não está relacionada apenas com a fase de destacamento do solo pela chuva mas, também, com os aspectos relacionados com a fase de transporte dos sedimentos via escoamento superficial difuso.

Analisando a Figura 2A, por exemplo, verifica-se que as variações das vazões ao longo do tempo foram pequenas, permanecendo estáveis até o final do experimento, enquanto a concentração de sedimentos em suspensão diminuiu. Se considerar que a vazão representa a capacidade de transporte, sugerir-se-á que o processo de erosão foi limitado pelo suprimento de sedimentos representado pela fase de destacamento do solo pela chuva. As Figuras 2B e 2C também mostram que os valores de vazão foram muito similares ao longo do tempo com capacidade de transporte semelhante. Entretanto, verifica-se que os valores de CSS não mostraram redução, como observado para o tratamento PC (Figura 2A). Outra constatação foi que a ordem de grandeza da CSS do tratamento MN (Figura 2C) foi inferior à do tratamento PD (Figura 2B). Supondo-se, então, que os tratamentos MN e PD apresentaram resistência a desagregação semelhante, conforme indica o IEA, e uma mesma capacidade de transporte (verificada pela semelhança entre as vazões), as diferenças entre esses tratamentos poderiam ser atribuídas à maior dissipação de energia (verificada através do fator $f$ ) que ocorreu no tratamento $\mathrm{MN}$ e que, por sua vez, afetou o transporte dos sedimentos. A 
menor erosão em entressulcos, verificada no tratamento $\mathrm{MN}$, se deve não somente à maior coesividade do solo, mas também a fatores hidráulicos relacionados com a fase de transporte de sedimentos. Em virtude do tratamento $\mathrm{MN}$ apresentar uma perda maior de carga, medida pelo fator $f$, sugere-se que o escoamento necessitou desprender mais energia para vencer as forças de resistência do escoamento em detrimento da energia que poderia ter sido utilizada para o transporte dos sedimentos. Esta condição poderia ser interpretada como um processo interativo entre a resistência a desagregação provocada pelo impacto das gotas da chuva e a capacidade de transporte de sedimentos. Em situações em que os agregados superficiais resistem a desagregação, é criada uma condição de maior rugosidade superficial que, por sua vez, altera a hidráulica do escoamento no sentido de diminuir a capacidade de transporte de sedimentos pelo escoamento. Então, na análise da maior ou menor suscetibilidade ao processo erosivo em entressulcos não devem ser examinados só os fatores intrínsecos do solo, mas, sim, todos os fatores capazes de contemplar as duas etapas do processo erosivo que são a desagregação e o transporte.

\section{ConclusõEs}

1. O tratamento MN necessitou do dobro de energia para desagregar e transportar a mesma quantidade de solo que os tratamentos PC e PD, condição explicada pela maior estabilidade dos agregados e rugosidade superficial.

2. O solo submetido ao tratamento PD apresentou uma estabilidade maior dos seus agregados, quando comparado com o tratamento $\mathrm{PC}$, sendo tal estabilidade atribuída à maior saturação de bases aliada à condição de maior adensamento desse solo.

3. A resistência ao processo de erosão em entressulcos está relacionada não apenas com as condições de coesividade do solo mas também a fatores relacionados com a fase de transporte dos sedimentos.

\section{LITERATURA CITADA}

Abrahams, A.; Persons, A. J. Resistence to overland flow on desert pavement and its implications for sediment transpor modeling. Water Resources Research, v.27, p.1827-1836, 1991.

Albuquerque, J. A.; Cassol, E. A.; Reinert, D. J. Relação entre erodibilidade entressulcos e estabilidade de agregados. Revista Brasileira de Ciência do Solo, v.24, p.141-151, 2000.

Aratani, R. G.; Freddi, O. S.; Centurion, J. F.; Andrioli, I. Qualidade física de um Latossolo vermelho acriférrico sob diferentes sistemas de manejo. Revista Brasileira de Ciência do Solo, v.33, p.677-687, 2009.

Bohn, H. L.; Mcneal, B. L.; O‘Connor, G. A. Soil chemistry. New York.: John Wiley \& Sons, 1995. 267p.

Bryan, R. B. Processes and significance of rill development. Catena Vertlag, Cremlingen-Destedt, v.8, p.1-15, 1987.
Chow, V. T. Open channel hydraulics, New York: McGraw-Hill Book Co., 1959. 680p.

Davis, J. G.; Waskom, R. M.; Bauder, T. A.; Cardon, G. E. Managing sodic soils . http://www.ext.colostate.edu/PUBS/ crops/00504.html. 25 Set. 2009.

Dingle, N. A.; Lee, Y. Terminal fallspeed of raindrops. Journal of Applied Meteorology, v.11, p.877-879, 1972.

Dontsova, K.; Norton, L. D. Effects of exchangeable Ca:Mg ratio on soil clay flocculation, infiltration and erosion In: Stott, D. E.; Steinhardt, G. C. (ed.). Sustaining the global farm. In: International Soil Conservation Organization Meeting, 10, Proceedings, 2001. p.24-29.

EMBRAPA - Empresa Brasileira de Pesquisa Agropecuária. Centro Nacional de Pesquisa de Solos (RJ). Manual de métodos de análise de solo. 2.ed. Rio de Janeiro: Embrapa CNPS, 1997.212p.

FEPAM-RS - Fundação Estadual de Proteção Ambiental Henrique Luis Roessler. Biodiversidade do Rio Grande do Sul. http://www.fepam.rs.gov.br. 5 Abr. 2008.

Foster, G. R. Modeling the erosion process. In: Hann, C. T.; Johnson, H. P.; Brakensiek, D. L. (ed.) Hydrologic modeling of small watersheds. St. Joseph: American Society of Agricultural Engineering, 1982. p.297-380.

Grissenger, E. H. Resistance of selected clays system to erosion by water. Water Resources Research, v.2, p.131-138. 1966.

Gunn, R.; Kinzer, G. D. The terminal velocity of fall for water droplets in stagnant air. Journal Meteorology, v.6, p.243$248,1949$.

Heil, J. W.; Juo, A. S. R.; Mcinnes, K. Soil properties influencing surface sealing of some sandy soils in the Sahel. Soil Science, v.162, p.459-469, 1997.

Hillel, D. Introduction to soil physics. New York: Academic Press, 2003. 364p.

Jacobs, A.; Rauber, R.; Ludwing, B. Impact of reduced tillage on carbon and nitrogen storage of two Haplic Luvisols after 40 years. Soil and Tillage Research, v.102, p.158-164, 2009.

Katz, D. M.; Watts, F. J.; Burroughs, E. R. Effects of surface roughness and rainfall impact on overland flow. Journal Hydrology Division , v.121, p.546-553, 1995.

Kemper, W. D.; Chepil, W. S. Size distribution of aggregates. In: Black, C. A. (ed.) Methods of soil analysis, physical and mineralogical properties, including statistics, measurement and sampling. Madison: American Society of Agronomy, p.499-510, 1965.

Kinnel, P. I. A. The effect of flow depth on sediment transport induced by raindrops impacting shallow flows. Transactions of the American Society of Agricultural Enginners, v.34, p.161-168, 1991.

Kinnel, P. I. A. Particle travel distances and bed sediment compositions associated with rain-impacted flows. Earth Surface Processes and Landform, v.26, p.749-758 2003.

Kinnel, P. I. A. Sediment transport by medium to large raindrops impacting flows and subterminal velocity. Soil Science Society of America, v.69, p.902-905. 2005.

Kinnel, P. I. A. Simulations demonstrating interaction between coarse and fine sediment loads in rain-impacted flow. Earth Surface Processes, v.31, p.355-367, 2006. 
Levy, G. J.; Levin, J.; Shainberg, I. Seal formation and interrill soil erosion. Soil Science Society American Journal, v.58, p.203-209, 1994.

Line, D. E.; Meyer, L. D. Evaluating interrill and rill erodibilities for soils of different textures. Transactions of the American Society of Agricultural Engineers, v.32, p.1995-1999, 1989.

Melissa, A. S. S.; Mafra, A. L.; Albuquerque, J. A.; Rosa, J. D.; Bayer, C.; Mielniczuk, J. Propriedades físicas e teor de carbono orgânico de um Argissolo vermelho sob distintos sistemas de uso e manejo. Revista Brasileira de Ciência do Solo, v.31, p.329-337, 2006.

Mello, N. A. Efeito do sistema de manejo nos atributos do solo, movimentação de sedimentos e exportação de carbono orgânico numa microbacia rural sob cultura do fumo. Porto Alegre: UFRGS, 2006. 248p. Tese Doutorado

Merten, G. H.; Minella, J. P. G. Impact on sediment yield caused by intensification of tobacco production in a catchment in southern Brazil. In: Sediment Budgets 2.ed.Wallingford: IAHS Press, v. 291, p. 239-244, 2005.

Merten, G. H.; Nearing, M. A.; Borges, A. L. O. Effect of sediment load on soil detachment and deposition in rills. Soil Science Society of America Journal, v.65, p.861-868, 2001.

Meyer, L. D.; Foster, D. R.; Römkens, M. J. M. Source of soil eroded by water from upland slopes. In: Sediment Yield Workshop. Oxford. Proceedings. Washington: USDA, p.177$189,1975$.

Meyer, L. D.; Harmon, W. C. Multiple intensity rainfall simulator for erosion research on row sideslopes. Transactions of the American Society of Agricultural Engineering, v.22, p.100103, 1979.

Minella, J. P. G.; Merten, G. H.; Reichert, J. M.; Santos, D. R. Identificação e implicações para a conservação do solo das fontes de sedimentos em bacias hidrográficas. Revista Brasileira de Ciência do Solo, v.31, p.1637-1646, 2007.

Minella, J. P. G.; Walling, D.; Merten, G. H. Combining sediment source tracing techniques with traditional monitoring to assess the impact of improved land management on catchment sediment yields. Journal of Hydrology, v.348, p.546-563, 2008.

Morgan, R. P. C. Soil erosion and conservation. 2.ed. Oxford: Blackwell Publishing, 1995. 198p.

Nunes, M. C. M.; Cassol, E. A. Estimativa da erodibilidade em entressulcos de Latossolos no Rio Grande do Sul. Revista Brasileira de Ciência do Solo, v.32, p.2839-2845, 2008.

Oades, J. M. The retentions of organic matter in soils. Biogeochemistry, Dordrecht, v.5, p.35-70, 1988.

Onweremadu, E. U.; Onyia, V. N.; Anikwe, M. A. N. Carbon and nitrogen distribution in water stable aggregates under two tillage techniques in Fluvisols of Werri area, southeaster Nigeria. Soil Tillage Research, v.97, p195-206. 2007.

Rauws, G.; Govers, G. Hydraulic and soil mechanical aspects of rill generation on agricultural soils. Journal of Soil Science, v.39, p111-124. 1988.

Razafimbelo, T. M;, Albrech, A.; Olive, R.; Chevallier, T.; Lardy, L. C.; Feller, C. Aggregate associated-C and physical protection in a tropical clayed soil under Malagasy conventional and no-tillage systems. Soil and Tillage Research, v.98, p.140-149, 2008.

Reichert, J. M.; Norton, L. D. Surface seal micromorphology as affected by fluidized bed combustion bottom-ash. Soil Technology, v.7, p.303-317, 1995.
Rockenbach, C. A.; Semmelmann, F. R. Comparação dos índices de energia cinética de uma chuva simulada obtidos através de um distrometer e de expressões derivadas de chuvas naturais. In: Simpósio Nacional de Controle de Erosão, 1998, Presidente Prudente. Resumos... Presidente Prudente: ABGE, 1998. p.86-87.

Rose, C. An introduction to the environmental physics of soil, water and watersheds. Cambridge: Cambridge University Press, 2004. 434p.

SAEG - Sistema para análises estatísticas. Versão 8.0. Viçosa: Fundação Arthur Bernardes, 2000.

Savat, J. Laboratory experiments on erosion and deposition of loess by laminar sheet flow and turbulent rill flow. In: Vogt, H.; Vogt, T. (ed.). Colloque sur 1'erosion agricole des sols en milieu tempere nom Mediterraneen. Strasbourg: 1' Universite Louis Pasteur. 1979. p.139-143.

Semmelmann, F. R. Determinação da energia cinética de chuvas simuladas. In: Simpósio Brasileiro de Recursos Hídricos, 4, 1991. Rio de Janeiro. Anais... Rio de Janeiro: ABRH, 1991. CD-Rom

Simansky, V.; Tobiasova, E.; Chipik, J. Soil tillage and fertilization of Orthic Luvisol and their influence on chemical proprieties, soil structure stability and carbon distribution in water-stable macro-aggregates. Soil and Tillage Research, v.100, p.125$132,2008$.

Simons, D. B.; Sentürk, F. Sediment transport technology: Water and sediment dynamics. Littleton: Water resources publications. 1992. 897p.

Singh, V. P. Analytical solutions of cinematic for erosion on a plane: II Rainfall of finite duration. Advanced Water Research, v.6, p.88-95, 1983.

Spera, S. T.; Denardin, J. E.; Escosteguy, P. A. V.; Santos, H. P.; Figueroa, E. A. Dispersão de argila em microagregados de solo incubado com calcário. Revista Brasileira de Ciência do Solo, v.32, p.2613-2620, 2008.

Streck, E. V.; Kampf, N.; Dalmolin, R. S.; Klant, E.; Nascimento, P. C.; Schneider, P.; Giasson, E.; Pinto, L. F. S. Solos do Rio Grande doSul. PortoAlegre: EMATER/RS-ASCAR, 2008. 222p.

Tedesco, M. J.; Gianello, C.; Bissani, C. A.; Bohnem, H.; Volkweiss, S. J. Análise de solo, plantas e outros materiais. 2.ed. Porto Alegre: Universidade Federal do Rio Grande do Sul, 1995. 174p. Boletim Técnico, 5

Tisdall, J. M.; Oades, J. M. Stabilization of soil aggregate by the root systems of ryegrass. Australian Journal Soil Research, v.17, p.429-441, 1979.

Toy, T. J.; Foster, G. R.; Renard, K. G. Soil erosion: processes, prediction, measurement, and control. New York: John Wiley \& Sons, 2002.337p.

Veiga, M.; Reinert, D. J.; Reichert, J. M. Aggregate stability as affected by short and long-term tillage systems and nutrient sources of Hapludox in southern Brasil. Revista Brasileira Ciência do Solo, v.33, p.767-777, 2009.

Woolhiser, D. A.; Liggett, J. A. Unsteady onedimensional flow over a plane - The rising hydrograph. Water Resources Research, v.3, p.753-771, 1967.

Yoder, R. E. A direct method of aggregate analysis of soil and a study of the physical nature of erosion losses. Journal of American Society of Agronomy, v.28, p.337-357, 1936.

Young, R. A.; Wiersma, J. L. The role of rainfall impact in soil detachment and transport. Water Resources Research, v.9, p.1629-1639, 1973. 